\title{
La Rieducazione Posturale Globale nelle patologie muscolo-scheletriche: evidenze scientifiche e indicazioni cliniche
}

\author{
Rééducation Posturale Globale in musculoskeletal diseases: \\ scientific evidence and clinical practice
}

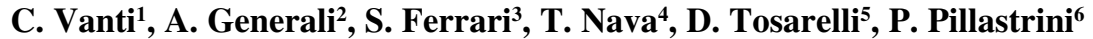 \\ ${ }^{1}$ Corso di Laurea in Fisioterapia, Università di Bologna; ${ }^{2}$ Fisioterapista, Bologna; ${ }^{3}$ Scuola Italiana di Riabilitazione Integrata (S.I.R.I.); \\ ${ }^{4}$ Fisioterapista, Sesto San Giovanni (MI); ${ }^{5}$ Istituti Ortopedici Rizzoli, Bologna; \\ ${ }^{6}$ Dipartimento di Scienze Neurologiche, Università di Bologna
}

\begin{abstract}
SUMMARY
Several studies on the treatment of musculoskeletal diseases with physiotherapy and clinical experiences on the basis of a method called Rééducation Posturale Globale (RPG), have highlighted the usefulness of this treatment. Although such treatment technique is commonly used in physical therapy practice, only few studies support its therapeutic effectiveness.

Objective: To search the literature for evidence of RPG effectiveness, in order to identify the most appropriate therapeutic contexts for its use.

Methods: A review of the literature through the following databases: PubMed, Embase, Cinahl, Pedro, and Medscape. The keywords used for the search in the databases are: Rééducation Posturale Globale, Souchard, Posture, and Manual Therapy. The following clinical studies were selected: randomized controlled studies, non-randomized controlled studies, observation studies, and case reports, in English, Spanish, Portuguese, and Italian.

Results: Out of 18 studies found, 9 were analyzed: 2 randomized controlled studies, 2 non-randomized controlled studies, 3 non-controlled studies, and 2 case reports.

Conclusions: The RPG method has been shown to be an effective treatment technique for musculoskeletal diseases, in particular for ankylosing spondylitis, acute and chronic low back pain, and lumbar discherniation. Although the scarcity of rigorous experimental trials on a large scale does not allow the drawing of undisputable conclusions, the results gathered up to now are an encouragement to carry on research in the field of conservative treatment.
\end{abstract}

Reumatismo, 2007; 59(3):192-201

\section{INTRODUZIONE}

N umerosi studi clinici hanno evidenziato l'utilità della fisiochinesiterapia nelle patologie muscolo-scheletriche e reumatiche: è risultata efficace per il dolore e la funzione articolare nell' artrosi del ginocchio (1) e dell'anca (2), nella spondilite anchilosante $(3,4)$, nell' artrite reumatoide

Indirizzo per la corrispondenza:

Prof. Pillastrini Paolo

Dipartimento di Scienze Neurologiche

Via Ugo Foscolo, 7

40123 Bologna

E-mail: paolo.pillastrini@unibo.it
$(2,5-8)$, nella diminuzione della massa ossea ( 9 , 10), nella prevenzione dei crolli vertebrali (11) e del rischio di caduta nell'osteoporosi (12-15). Seppur in un contesto che riconosce l'utilità della fisioterapia e dell'attività fisica, possono essere adottate strategie riabilitative differenti in funzione della patologia, della fase, degli obiettivi terapeutici e delle condizioni individuali. In tutti i casi, è necessario un progetto riabilitativo globale. La fisioterapia deve saper intervenire sul dolore e cercare di rallentare l'evoluzione sistemica degenerativa primaria, ma deve agire anche negli esiti del danno secondario, nelle condizioni disfunzionali favorenti la sintomatologia, nei fattori di rischio di ri- 
caduta e nelle complicanze psicologiche, al fine di massimizzare le funzioni e minimizzare la disabilità e l'handicap (16). Esercizi specifici per migliorare la forza muscolare, la mobilità e la stabilità articolare, la disfunzione sensoriale che si riflette nella diminuzione di propriocezione, costituiscono il core dell'intervento riabilitativo. Inoltre è essenziale educare il paziente sulla gestione dell'ansia, sulla necessità di periodi di riposo e sull'importanza del rilassamento e dell'economia articolare $(17,18)$. Uno specifico metodo di fisioterapia, denominato Rieducazione Posturale Globale (RPG) è stato sviluppato da Philippe Emmanuel Souchard dopo anni di esperienza clinica e può essere particolarmente indicato, per le sue caratteristiche, nel trattamento di alcune patologie muscolo-scheletriche e reumatiche $(3,4)$. Questo metodo consiste nel riequilibrare le tensioni miofasciali che possono essere responsabili del sovraccarico articolare, agendo sull'allungamento di muscoli antigravitari contratti o retratti all'interno di differenti catene muscolari statiche. Secondo Souchard (19), i muscoli con maggior percentuale di fibre toniche, di I tipo, sono sempre in contrazione, e vanno facilmente incontro a retrazioni ed accorciamenti, con riduzione dell' "elasticità" mio- fasciale e squilibri statici e dinamici persistenti. La RPG è un approccio globale, che ricerca la causa biomeccanica del problema anche in distretti adiacenti e coinvolge il paziente attivamente nel cambiamento posturale e gestuale. Si applica nelle anomalie strutturali (scoliosi, iperlordosi, ipercifosi, ginocchia vare o valghe, piedi piatti o cavi), nelle disfunzioni articolari, nei deficit respiratori (escursione toracica e diaframmatica, frequenza e ritmo ventilatorio), nelle patologie sportive, ecc. I principi della RPG sono:

- causalità: ogni problema muscoloscheletrico dipende da una causa; durante le sedute il fisioterapista cerca, attraverso le catene muscolari, di risalire alla causa partendo dall'effetto;

- globalità: non si può trattare una struttura osteomuscolare analiticamente, senza tenere conto delle influenze e della interdipendenza con i distretti contigui, della globalità dell'individuo e degli aspetti psico-motivazionali;

- individualità: non si tratta la malattia, ma la disabilità dell'individuo, con le sue peculiarità e caratteristiche. Ogni trattamento è quindi unico ed in questo la RPG si differenzia nettamente dalle metodiche classiche.

La RPG è considerata "ginnastica dolce", adatta

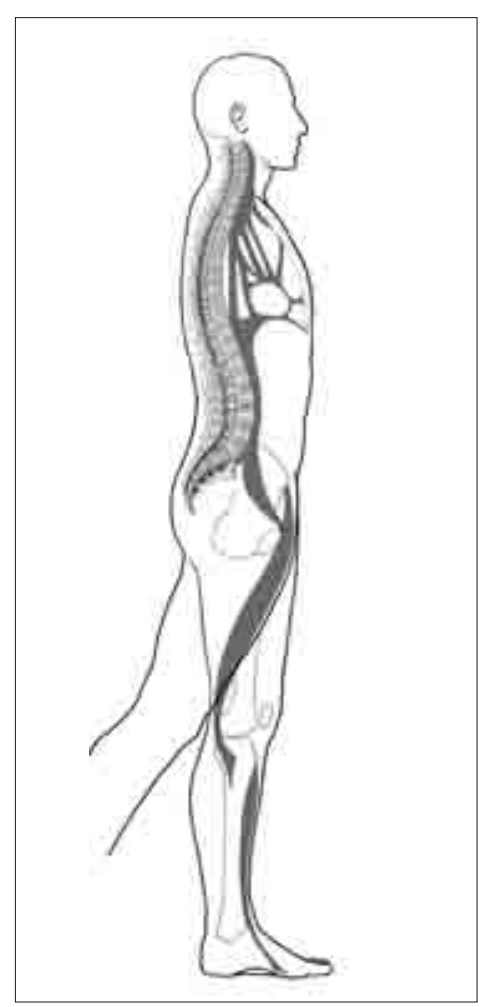

Figura 1 - Grande catena anteriore.

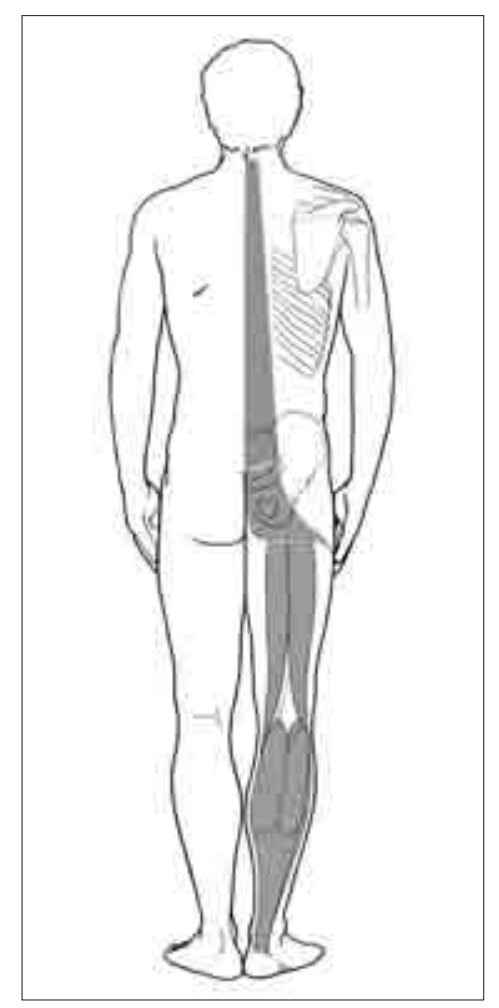

Figura 2 - Grande catena posteriore.

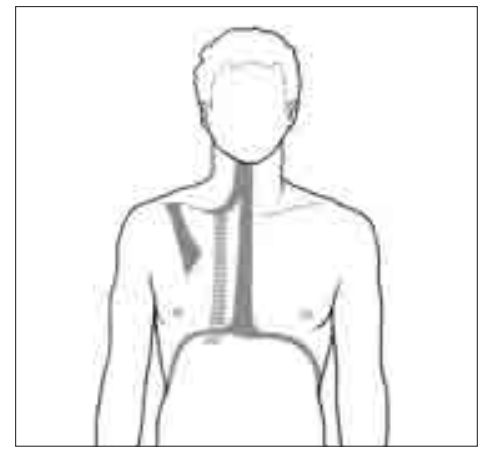

Figura 3 - Catena inspiratoria.

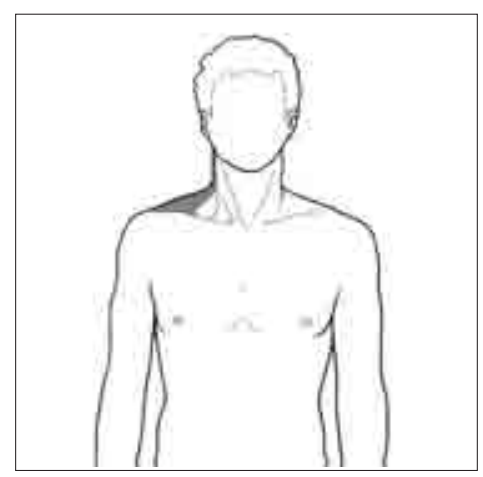

Figura 4 - Catena superiore della spalla. 
quindi anche agli adolescenti ed alle persone anziane e consiste in posture di allungamento attivo di specifiche catene muscolari, progressive, che si sviluppano in piedi, da seduto o al suolo. Le catene muscolari statiche sulle quali indirizzare il trattamento sono principalmente due:

- la grande catena anteriore - è una catena miofasciale formata dal sistema sospensore del diaframma e dei visceri, lo sterno-cleido-mastoideo, il muscolo lungo del collo, gli scaleni, i pilastri del diaframma, l'ileo-psoas e la fascia iliaca, gli adduttori pubici e il tibiale anteriore (Fig. 1);

- la grande catena posteriore - è una catena miofasciale formata dai muscoli spinali, i pelvi-tro- canterici, il grande gluteo, gli ischio-crurali, il popliteo, il tricipite surale e i muscoli plantari (Fig. 2).

Ad esse si aggiungono, in relazioni diverse, alcune catene muscolari accessorie: inspiratoria (Fig. 3), superiore della spalla (Fig. 4), antero-interna della spalla (Fig. 5), anteriore del braccio (Fig. 6), antero-interna dell'anca (Fig. 7), laterale dell'an$c a$ (Fig. 8). Il lavoro attivo è il requisito indispensabile affinché si possa realizzare il "rilasciamento tonico" dei muscoli accorciati sfruttando il riflesso miotatico inverso e tutte le correzioni possano venire integrate dai meccanismi automatici deputati al controllo della postura e della miglior

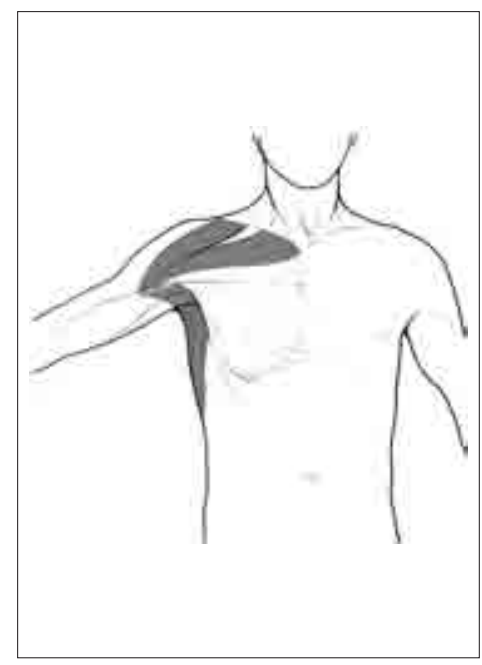

Figura 5 - Catena antero-interna della spalla.

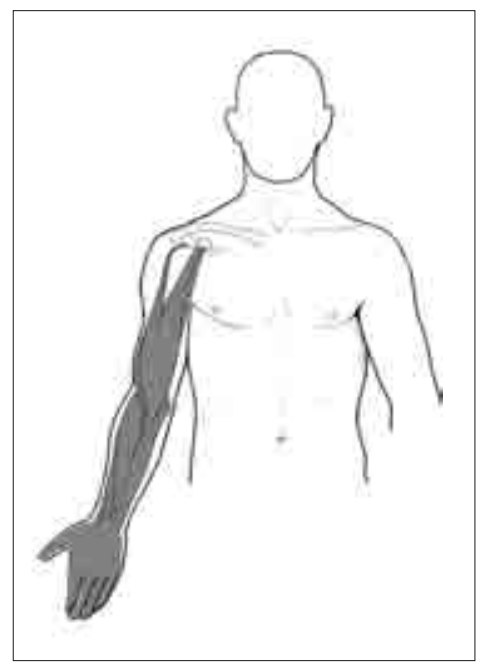

Figura 6 - Catena anteriore del braccio.

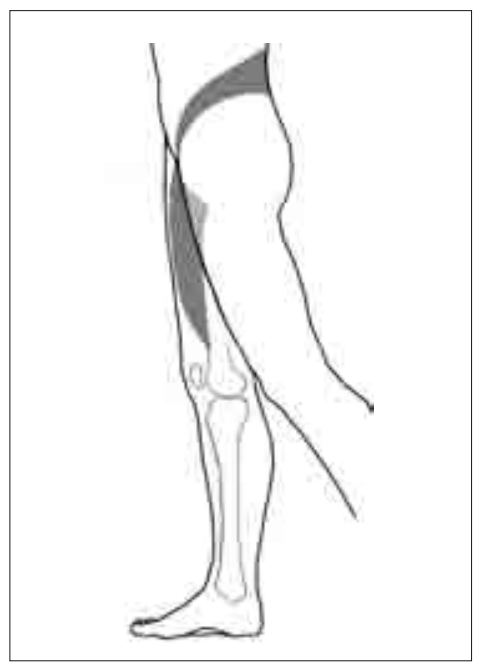

Figura 7 - Catena antero-interna dell'anca.

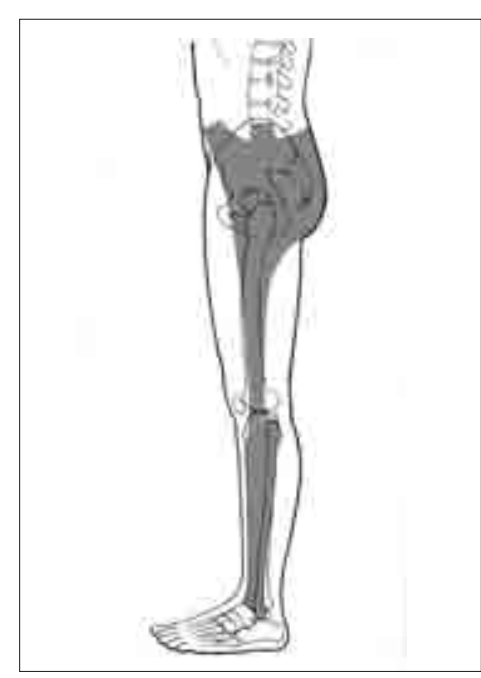

Figura 8 - Catena laterale dell'anca.

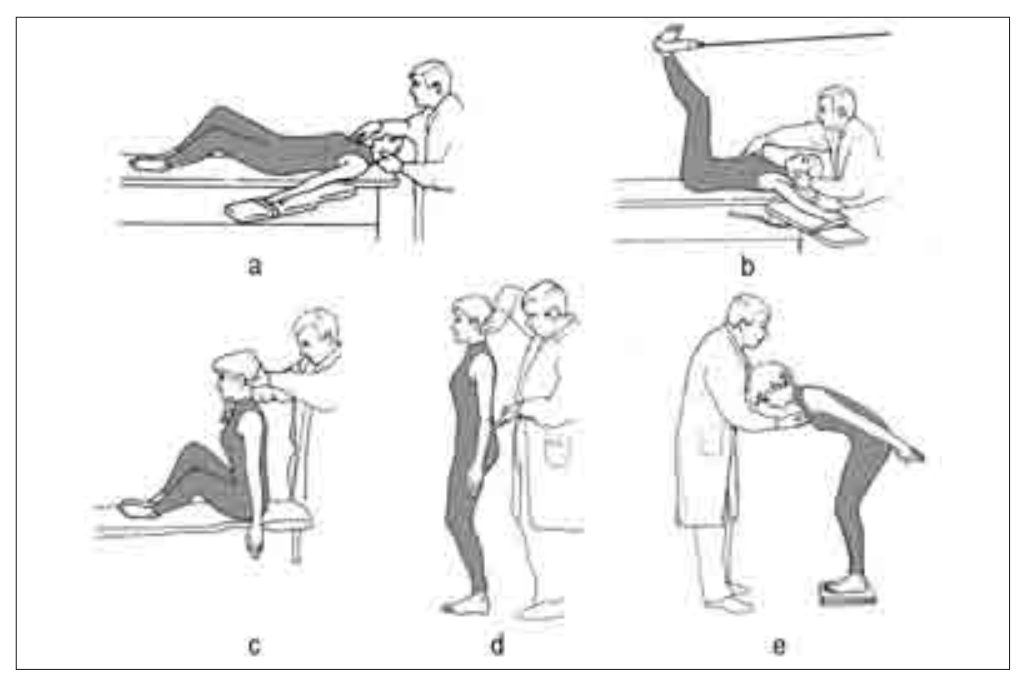

Figura 9 - a) Postura "rana al suolo". b) Postura "rana in aria". c) Postura seduta. d) Postura in piedi. e) Postura in piedi, con busto inclinato in avanti. 
gestualità. La ricerca delle correzioni deve avvenire coscientemente, senza dolore o con un minimo dolore facilmente gestibile dal paziente, attraverso interventi anche manuali, per favorire l'allungamento, nel senso longitudinale, dei muscoli contratti o retratti all'interno delle catene muscolari accorciate. A titolo di esempio, la protrusione del capo e l'aumento della cifosi toracica possono dipendere dall'accorciamento della catena anteriore, la protrazione delle spalle e la loro rotazione interna sono causate dall'accorciamento della catena antero-interna della spalla, la flessione e la rotazione interna dell'anca sono provocate dall'accorciamento della catena antero-interna dell' anca. Le posture a disposizione sono diverse, scelte opportunamente per poter affrontare le molteplici condizioni della patologia e del paziente (Fig. 9). Malgrado questa tecnica sia molto utilizzata dai fisioterapisti, poche ricerche ne supportano l'efficacia terapeutica, che pare confermata principalmente dall'opinione di autori e dalle singole esperienze cliniche. Scopo di questo lavoro è quello di ricercare in letteratura le evidenze disponibili sulla RPG, con l'obiettivo di valutarne l'effettiva efficacia ed individuare i contesti terapeutici più idonei al suo impiego.

\section{MATERIALI E METODI}

Nel periodo tra Giugno e Ottobre 2006, due revisori indipendenti (AG e CV) hanno condotto una revisione della letteratura inerente l'applicazione clinica della RPG. Sono state consultate inizialmente le seguenti banche dati: PubMed, Embase, Cinahl, Pedro, utilizzando le parole chiave: Rééducation Posturale Globale, Souchard, Posture, Manual Therapy. Data la scarsa numerosità dei lavori scientifici reperiti, la ricerca è stata ampliata utilizzando la bibliografia degli articoli per risalire ad ulteriori pubblicazioni inerenti l'argomento e ricorrendo ad altre Banche Dati (ad es. Medscape) e a siti web (ad es. /www.rpg-souchard.com; www.bioline.org.br; www.itg.rpg.org; www.actafisiatrica.org.br, www.reeducacaoposturalglobal. hpg.com.br).

Attraverso "Index Medicus - Locator plus", è stata comunque verificata l'indicizzazione delle riviste in cui gli articoli erano stati pubblicati.. Sono stati selezionati studi clinici randomizzati controllati, non randomizzati controllati, studi osservazionali e case report, in lingua inglese, spagnola, portoghese e italiana.

\section{RISULTATI}

Nella letteratura internazionale sono stati trovati 18 studi sull'efficacia clinica della RPG; 4 di essi sono stati esclusi dalla revisione perché non pertinenti o di scarso valore. Altri 5 sono stati successivamente esclusi perché non riguardanti prevalentemente le patologie muscolo-scheletriche: 2 sulle disfunzioni respiratorie $(20,21), 1$ sui disturbi del post-partum (22), 1 sulla pressione plantare e la posizione del centro di gravità (23) e 1 sulla forza muscolare dei flessori ed estensori di ginocchio (24). Dei 9 studi analizzati, 2 sono randomizzati controllati, 2 sono non randomizzati controllati, 3 sono non controllati e 2 sono case report. Gli studi sono stati condotti in diversi campi di applicazione della RPG: 2 sulla spondilite anchilosante, 2 sulla lombalgia, 1 sulla lombosciatalgia, 2 sull'ernia discale, 2 su alterazioni posturali dolorose del rachide. Per facilitarne l'interpretazione, $i$ risultati dei 9 studi sono stati riportati in sintesi e ordinati per campo di applicazione. Nella tabella I ne sono stati quindi analizzati caratteristiche principali, tipi di trattamento e risultati $(3,4,25-31)$ (Tab. I).

\section{DISCUSSIONE E CONCLUSIONI}

Questa revisione, anche se include un numero esiguo di studi, evidenzia che la RPG è un'interessante metodologia per il trattamento delle patologie muscolo-scheletriche. L'outcome positivo riguarda sia parametri clinici (dolore, postura, mobilità articolare), sia indici di funzionalità e/o disabilità (Owestry Disability Index, Autonomia nelle ADL).

Dalla revisione della letteratura si possono trarre alcune conclusioni generali, in cui il livello di evidenza (Tab. II) è stato riportato in relazione al numero e alla tipologia degli studi pubblicati (Tab. III).

Nei 4 studi controllati, i gruppi sperimentali sottoposti a trattamenti basati sul metodo RPG hanno ottenuto miglioramenti superiori rispetto ai gruppi di controllo, trattati con fisioterapia tradizionale o riposo. Gli studi clinici non controllati e i case report hanno dato risultati positivi, ma la significatività di tali pubblicazioni ed il conseguente livello di evidenza non si possono ritenere di alto livello. Tra i 9 studi analizzati, quelli meglio condotti sono i due RCT relativi alla spondilite anchilosante $(3,4)$, in cui è stato dimostrato che i pa- 


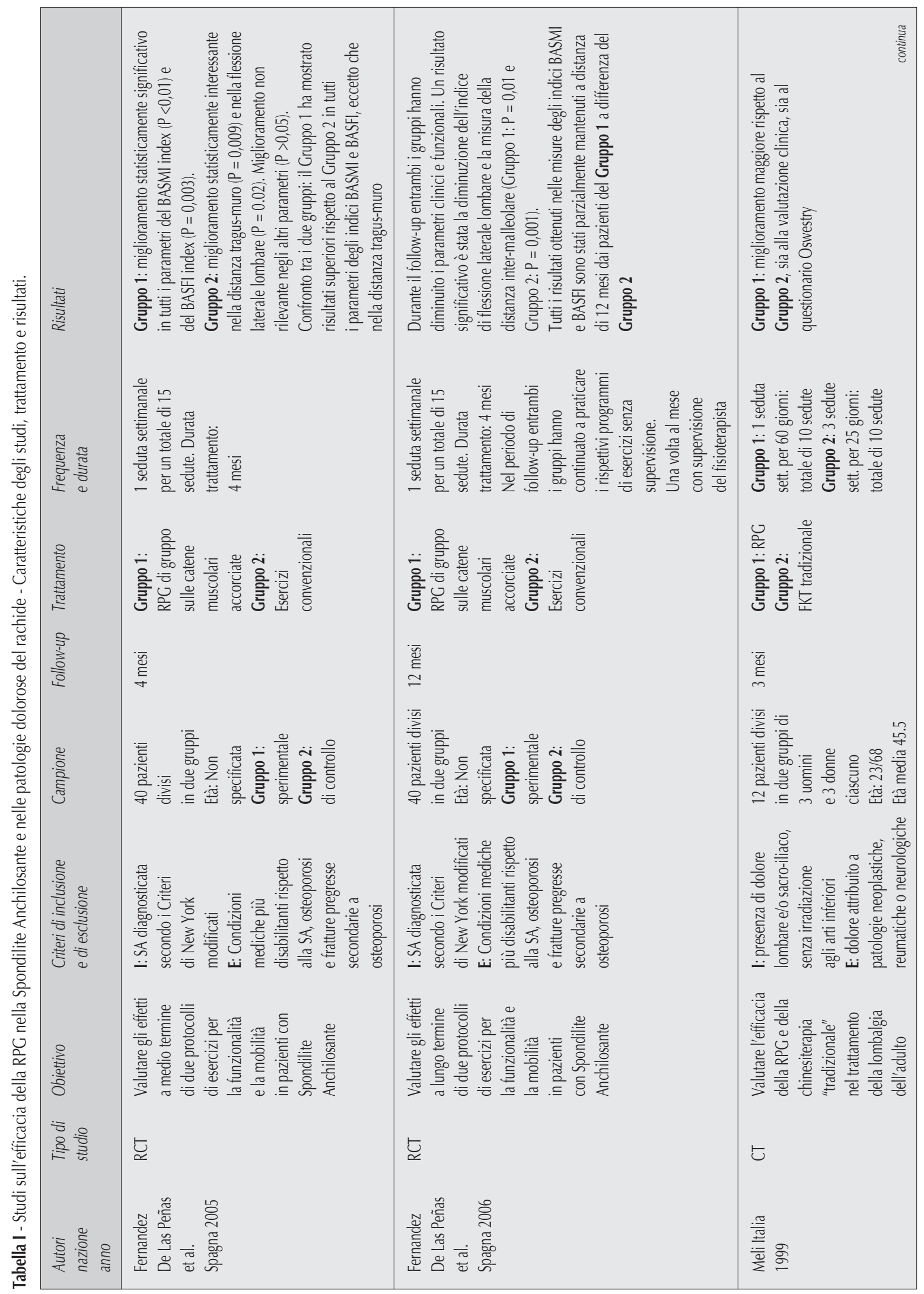




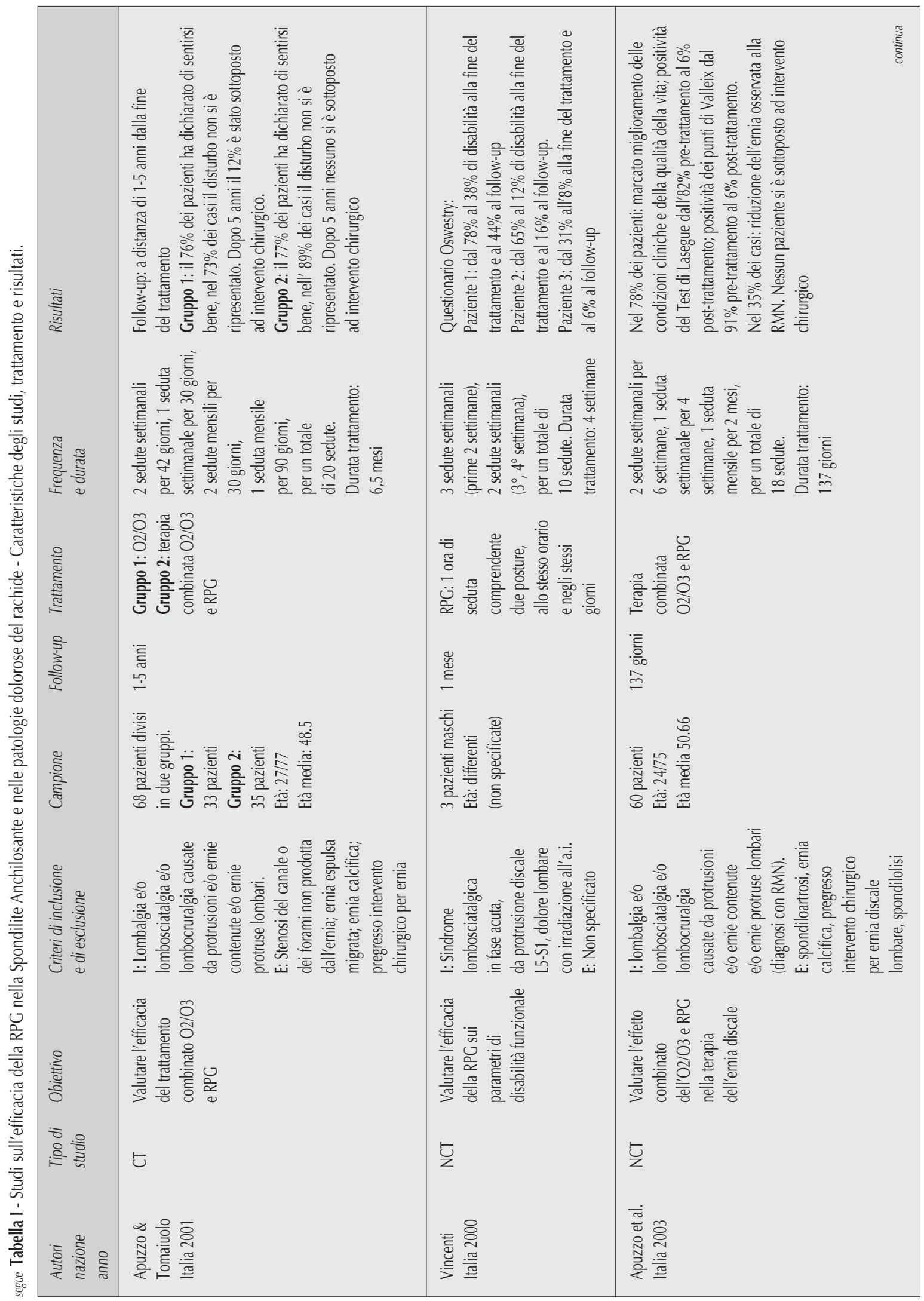




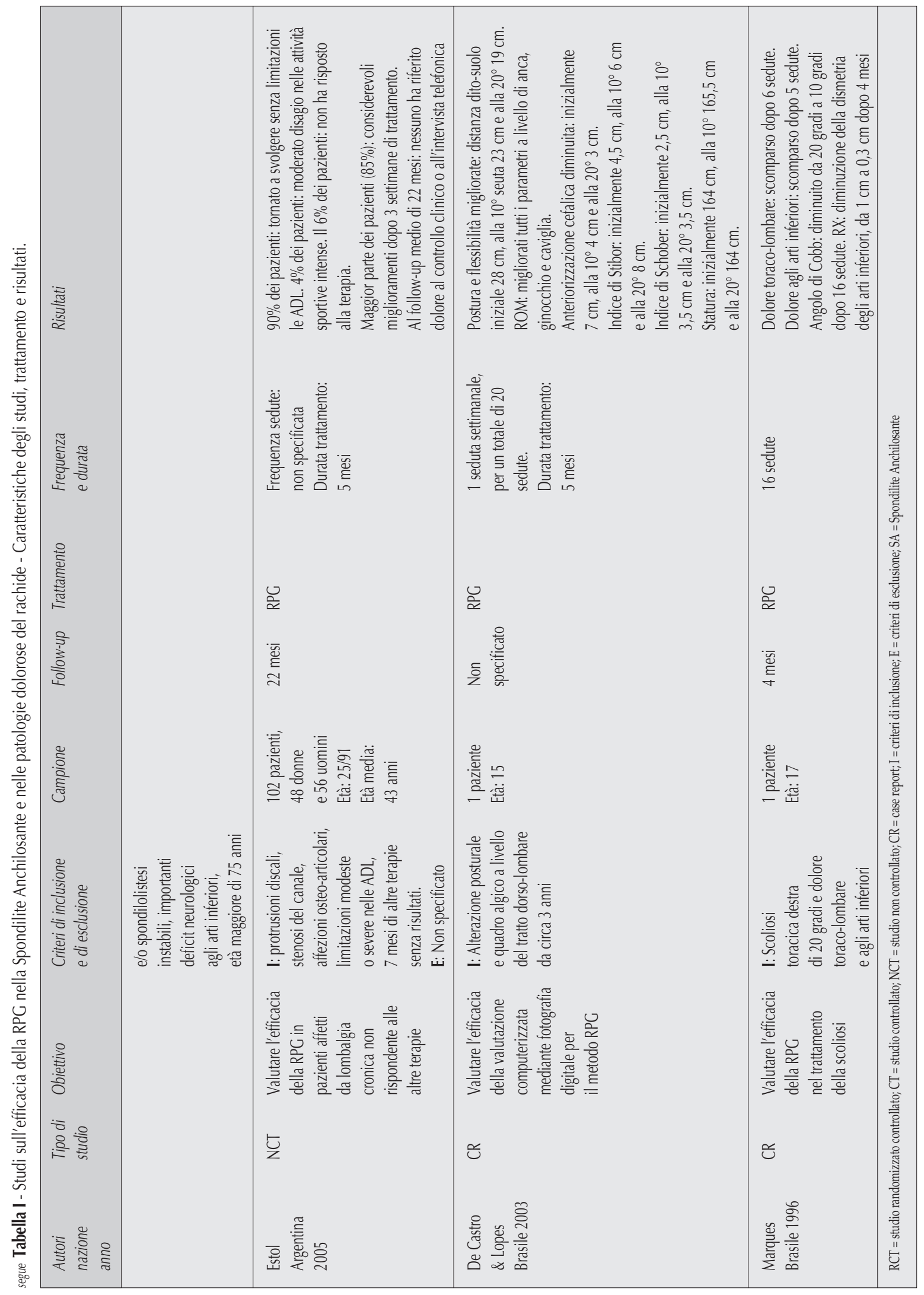


Tabella II - Livelli di evidenza utilizzati per attribuire la rilevanza scientifica agli studi analizzati.

\begin{tabular}{|ll|}
\hline $\begin{array}{l}\text { Livello } \\
\text { di evidenza }\end{array}$ & Base dell'evidenza \\
\hline $1 \mathrm{a}$ & $\begin{array}{l}\text { Revisione sistematica (con omogeneità) } \\
\text { di svariati RCT }\end{array}$ \\
\hline $1 \mathrm{~b}$ & Singoli RCT \\
\hline $2 \mathrm{a}$ & $\begin{array}{l}\text { Revisione sistematica (con omogeneità) } \\
\text { di studi di coorte }\end{array}$ \\
\hline $2 \mathrm{~b}$ & Singoli studi di coorte \\
\hline $2 \mathrm{c}$ & Studi di outcome \\
\hline $3 \mathrm{a}$ & $\begin{array}{l}\text { Revisione sistematica (con omogeneità) } \\
\text { di studi caso-controllo }\end{array}$ \\
\hline $3 \mathrm{~b}$ & Singoli studi caso-controllo \\
\hline 4 & $\begin{array}{l}\text { Serie di casi (e studi di coorte } \\
\text { e caso-controllo di bassa qualità) }\end{array}$ \\
\hline 5 & \begin{tabular}{l} 
Opinione di esperti \\
\hline
\end{tabular} \\
\hline
\end{tabular}

zienti trattati con la RPG hanno raggiunto risultati migliori in tutte le misure degli indici BASMI e BASFI, sia a breve termine (dopo un periodo di trattamento di 4 mesi), sia a lungo termine (al follow-up di 12 mesi), rispetto ai pazienti del gruppo di controllo, sottoposti ad un programma di fisioterapia convenzionale basato su stretching analitici ed esercizi di mobilizzazione. Un approccio globale pare quindi più efficace per i pazienti con spondilite anchilosante, rispetto ad esercizi analitici, come è stato riportato anche da una recente re- visione sistematica (32). Anche nella lombalgia e nalla lombosciatalgia acuta, la RPG ha evidenziato positivi risultati rispetto alla funzionalità valutata con il Questionario Oswestry $(25,27)$, pure in confronto alla fisiochinesiterapia analitica convenzionale (25). In un ampio campione di soggetti con lombalgia cronica non rispondente alle altre terapie, la RPG ha dato positivi risultati sia rispetto al recupero delle ADL, che in relazione al dolore (29). In pazienti con ernia discale, la RPG associata alla terapia $\mathrm{O} 2-\mathrm{O} 3$ ha mostrato apportare risultati migliori rispetto alla riduzione della sintomatologia e al ricorso alla chirurgia, in confronto alla sola terapia $\mathrm{O} 2-\mathrm{O} 3(26,28)$. Alcuni case reports hanno inoltre evidenziato che in pazienti con alterazione posturale e dolore la RPG risulta efficace nel migliorare la postura e ridurre la sintomatologia $(30,31)$, anche se il livello di evidenza di tali lavori non è tale da poter dimostrare in modo significativo una simile conclusione. In base a questa revisione della letteratura, le indicazioni terapeutiche dell'RPG si possono quindi rivolgere al trattamento conservativo di alcune patologie muscolo-scheletriche e reumatiche, sia in fase acuta, sia in fase cronica. Non siamo in grado di fornire ulteriori indicazioni rispetto all'applicazione della RPG in altre condizioni cliniche in cui tale tecnica potrebbe essere razionalmente indicata, quali ad esempio l'artrosi, la fibromialgia, o le condizioni dolorose del rachide cervicale o degli arti. Possiamo però fornire alcune indicazioni di massima rispetto all' applicazione di tale procedura. Le posture in scarico (Fig. 9a e 9b), più semplici e gestibili dal paziente, sono maggiormente indicate quando la sintomatologia dolorosa è ancora pre-

Tabella III - Tabella conclusiva dei risultati della revisione, in base ai livelli di evidenza adottati e indicati nella Tabella II.

\begin{tabular}{|lcc|}
\hline Conclusioni & Riferimenti bibliografici & Livello di evidenza \\
\hline $\begin{array}{l}\text { In pazienti con spondilite anchilosante, la RPG dà risultati migliori } \\
\text { rispetto agli indici BASMI e BASFI, in confronto alla fisioterapia } \\
\text { convenzionale }\end{array}$ & $(3,4)$ & $1 \mathrm{~b}$ \\
\hline $\begin{array}{l}\text { In pazienti con lombalgia, la RPG dà risultati migliori rispetto a dolore } \\
\text { e funzionalità, in confronto alla fisioterapia convenzionale }\end{array}$ & $(25,29)$ & $2 b$ \\
\hline $\begin{array}{l}\text { In pazienti con ernia discale, la RPG associata alla terapia O2-O3 } \\
\text { dà risultati migliori rispetto ai sintomi e al ricorso alla chirurgia, } \\
\text { in confronto alla sola terapia O2-O3 }\end{array}$ & $(26,28)$ & $2 b$ \\
\hline $\begin{array}{l}\text { In pazienti con lombosciatalgia, la RPG riduce la disabilità funzionale } \\
\text { In pazienti con alterazione posturale e dolore, la RPG migliora }\end{array}$ & $(27)$ & 4 \\
\hline la postura e riduce il dolore
\end{tabular}


sente, per cui le correzioni devono essere necessariamente fini e calibrate. Queste posture sono inoltre indicate quando è necessario agire sulle disfunzioni respiratorie (limitata escursione del torace). Le posture in carico (Fig. 9c, 9d, 9e), più complesse ma più funzionali, agiscono in maggior misura sulle retrazioni e sono preferibili nelle fasi asintomatiche, per la correzione dei maleallineamenti. La partecipazione attiva del paziente durante le posture è requisito indispensabile per l'effettuazione della tecnica, che quindi non può essere applicata nei bambini o negli anziani scarsamente collaboranti. Non è possibile standardizzare il numero e l'articolazione temporale delle sedute, anche se nella maggior parte degli studi, i soggetti sono stati sottoposti a due-tre sessoni settimanali di trattamento in fase acuta e a una sessione settimanale in fase subacuta e cronica, per un numero complessivo variabile da un minimo di 10 a un massimo di 20. Alcuni autori (26-28) hanno applicato una maggiore frequenza settimanale delle sedute all'inizio del trattamento, per progressivamente ridurne la frequenza. Il mantenimento del risultato della RPG nel tempo è stato confermato in tre studi $(4,26,29)$ anche con follow-up a lungo temine, dal minimo di 1 anno al massimo di 5 anni. Nessuno studio ha indagato l'effetto delle posture eseguite autonomamente a domicilio dopo addestramento da parte del fisioterapista (le cosiddette "autoposture"), anche se il razionale terapeutico suggerirebbe di consigliarle nei trattamenti a lungo termine, nell'ottica di ridurre i costi sanitari. L'attuale scarsità di trial sperimentali su larga scala e metodologicamente rigorosi non consente di trarre altre conclusioni , ma i risultati finora ottenuti rappresentano uno stimolo verso ulteriori ricerche in questo interessante settore del trattamento conservativo. In particolare, sarebbe interessante indagare con ulteriori studi randomizzati controllati l'efficacia della RPG nelle patologie oggetto di questa revisione ed ampliare la ricerca ad altre patologie reumatiche o ad altre sindromi dolorose del rachide e degli arti, confrontando i risultati di questa tecnica con quelli di altre procedure di fisiochinesiterapia.

\section{Ringraziamenti \\ Gli autori ringraziano Giliola Gamberini per il prezioso lavoro di preparazione delle immagini in- serite in questo articolo.}

\section{RIASSUNTO}

Numerosi trial clinici hanno evidenziato l'utilità della fisiochinesiterapia nelle patologie muscolo-scheletriche e reumatiche in particolare. Scopo dello studio è di ricercare le evidenze scientifiche disponibili su uno specifico metodo, denominato Rieducazione Posturale Globale (RPG). È stata condotta una revisione della letteratura nelle principali Banche Dati, con le Parole Chiave: Rééducation Posturale Globale, Souchard, Posture, Manual Therapy. Sono stati selezionati 9 studi clinici, che hanno evidenziato l'utilità della RPG per il trattamento di alcune patologie muscolo-scheletriche e reumatiche, in particolare nella Spondilite Anchilosante, nell'ernia discale e nella lombalgia acuta e cronica. La scarsità di trial sperimentali su larga scala e metodologicamente rigorosi non consente di trarre conclusioni certe, ma stimola ad ulteriori ricerche in questo ambito.

Parole chiave - Rieducazione, postura, patologie muscolo-scheletriche, terapia manuale, fisioterapia. Key words - Rehabilitation, posture, musculoskeletal diseases, manual therapy, physiotherapy.

\section{BIBLIOGRAFIA}

1. Punzi L, Canesi B, Carrabba M, Cimmino MA, Frizziero L, Lapadula G, et al. Italian consensus on Eular 2003 recommendations for the treatment of knee osteoarthritis. Reumatismo 2004; 56: 190-201.

2. Van Baar ME, Assendelft WJ, Dekker J, Oostendorp RA, Bijlsma JW. Effectiveness of exercise therapy in patients with osteoarthritis of the hip or knee: a systematic review of randomized clinical trials. Arthritis Rheum 1999; 42: 1361-9.

3. Fernandez-de-Las-Peñas C, Alonso-Blanco C, Morales-
Cabezas M, Miangolarra-Page JC. Two exercise interventions for the management of patients with ankylosing spondylitis: a randomized controlled trial. Am J Phys Med Rehabil 2005; 84: 407-19.

4. Fernandez-de-Las-Peñas C, Alonso-Blanco C, Alguacil-Diego IM, Miangolarra-Page JC. One-year follow-up of two exercise interventions for the management of patients with ankylosing spondylitis: a randomized controlled trial. Am J Phys Med Rehabil 2006; 85: 559-67.

5. Hidding A, Van Der Linden S, De Witte L. Therapeutic effects of individual physical therapy in Ankylos- 
ing Spondylitis related to duration of disease. Clin Rheumatol 1993; 12: 334-40.

6. Fransen M. When is physiotherapy appropriate? Best Pract Res Clin Rheumatol 2004; 18: 477-89.

7. Hammond A. Rehabilitation in rheumatoid arthritis: a critical review. Musculoskeletal Care 2004; 2: 135-51.

8. Dagfinrud H, Hagen KB, Kvien TK. (Cochrane Review). Physiotherapy interventions for ankylosing spondylitis. Cochrane Database Syst Rev 2005 (3).

9. Hartard M, Haber P, Ilieva D, Preisinger E, Seidl G, Huber J. Systematic strength training as a model of therapeutic intervention. A controlled trial in postmenopausal women with osteopenia. Am J Phys Med Rehabil 1996; 75: 21-8.

10. Preisinger E, Alacamlioglu Y, Pils K, Saradeth T, Schneider B. Therapeutic exercise in the prevention of bone loss. A controlled trial with women after menopause. Am J Phys Med Rehabil 1995; 74: 120-3.

11. Sinaki M, Itoi E, Wahner HW, Wollan P, Gelzcer R, Mullan BP, et al. Stronger back muscles reduce the incidence of vertebral fractures: a prospective 10 year follow-up of postmenopausal women. Bone 2002; 30: 836-41.

12. Wolff SL, Barnhart HX, Kutner NG, McNeely E, Coogler C, Xu T. Reducing frailty and falls in older persons: an investigation of Tai Chi and computerized balance training. Atlanta FICSIT Group. Frailty and Injuries: Cooperative Studies of Intervention Techniques. J Am Geriatr Soc 1996; 44: 489-97.

13. Lane JM, Nitpick M. Osteoporosis: current modes of prevention and treatment. J Am Acad Orthop Surg 1999; 7: 19-31.

14. Sinaki M, Brey RH, Hughes CA, Larson DR, Kaufman KR. Significant reduction in risk of falls and back pain in osteoporotic-kyphotic women through a Spinal Proprioceptive Extension Exercise Dynamic (SPEED) program. Mayo Clin Proc 2005; 80: 849-55.

15. Sinaki M, Brey RH, Hughes CA, Larson DR, Kaufman $\mathrm{KR}$. Balance disorder and increased risk of falls in osteoporosis and kyphosis: significance of kyphotic posture and muscle strength. Osteoporos Int 2005; 16 : 1004-10.

16. Nava T. La riabilitazione integrata delle malattie reumatiche. Ed. Masson: Milano, 2006.

17. Bryant GL, Cooley DA, Deal CL, Docken WP, Flood J, Schiaffino KM, et al. Guidelines for the Management of Rheumatoid Arthritis. Arthritis Rheum 1996; 39: 713-22.

18. Altman RD, Hochberg MC, Moskowitz RW, Schnitzer TJ. Recommendations for the Medical Management of Osteoarthritis of the hip and knee. Arthritis Rheum 2000; 43: 1905-15.

19. Souchard Ph. Basi del Metodo di Rieducazione Posturale Globale - Il Campo Chiuso. Ed. Marrapese: Roma, 1994.

20. Teodori RM, Moreno MA, Fiore Junior JF, Oliveira ACS. Alongamento da musculatura inspiratória por intermédio da reeducação postural global (RPG). Revi- sta Brasileira de Fisioterapia 2003; 7 : 25-30.

21. D’Ario L, Buonpensiero P. Funzione polmonare e postura nel trattamento integrato della Fibrosi Cistica con la Rieducazione Posturale Globale. Proceedings of the 5th International Congress of RPG; 2004 Oct 16-17; Rome. www.itg.rpg.org.

22. Santin C, Brunettin S. Esperienza di trattamento R.P.G. nel post-partum. Fondazione Internazionale di Rieducazione Posturale Globale - Monografia riservata ai soci. 2002; 3: 1-17.

23. Teodori RM, Guirro E, Santos RM. Plantar pressure distribution and center force location after postural global reeducation: a case relate. Fisioterapia em Movimento, Curitiba (Brazil) 2005;18: 27-35.

24. Ferrari S, Vanti C. Effetto di una procedura di allungamento attivo degli ischiocrurali sulla forza dei flessori e degli estensori del ginocchio. Scienza Riabilitativa 1996; 1: 51-3.

25. Meli O. Il rovescio della medaglia - Efficacia della Rieducazione Posturale Globale e della cinesiterapia "tradizionale" nel trattamento della lombalgia dell' adulto. Fondazione Internazionale di Rieducazione Posturale Globale - Monografia riservata ai soci. 2002; 3: 1-15.

26. Apuzzo D, Tomaiuolo A. Trattamento combinato Ossigeno-Ozono e Rieducazione Posturale Globale nell'ernia discale lombare: Studio follow-up. Eura Medicophys 2001; 37 (3 Suppl): 507-8. Proceedings of the National Congress S.I.M.F.E.R.; 2001; Rome.

27. Vincenti L. Studio sperimentale prospettico sull'efficacia del trattamento di Rieducazione Posturale Globale su pazienti lombalgici. Fondazione Internazionale di Rieducazione Posturale Globale - Monografia riservata ai soci. 2000; 2: 1-18.

28. Apuzzo D, Franzini M, Di Lucente L, Gioia MC, Cerasa A, Sabatini U. Combined effect of $\mathrm{O} 2 / \mathrm{O} 3$ and GPR in treatment of lumbar disc herniation: anatomical, clinical and patient-self-report evaluation. Eur J Clin Invest 2003; 33 (Suppl 1): 46.

29. Estol C. French physical therapy technique effective in patients with refractory chronic back pain. Proceedings of the 57th American Academy of Neurology's Annual Meeting; 2005 Apr 9-16; Miami Beach, Florida. www.medscape.com/viewarticle/503184 and www. parktribune.com.

30. De Castro PCG, Lopes JAF. Avaliação computadorizada por fotografia digital, como recurso de avaliação na Reeducação Postural Global. Acta Fisiatrica 2003;10:83-8, www.reeducacaoposturalglobal.hpg. com.br.

31. Marques AP. Escoliose tratada com Reeducação Postural Global. Relato de Caso. Revista Brasileira de Fisioterapia, Universidade São Paulo (Brazil) 1996; 3 : 65-8.

32. Ribeiro F, Leite M, Silva F, Sousa O. Exercício físico no tratamento da Espondilite Anquilosante: uma revisão sistemática. Acta Reumatológica Portuguesa 2007; 2: 129-37. 